\title{
A novel design of an isochronous integration [iIntegration] framework for first/second order multidisciplinary transient systems
}

\begin{abstract}
Of fundamental interest are multidisciplinary interactions encompassing: (1) first order systems such as those encountered in parabolic heat conduction, first order hyperbolic systems such as fluid flow, and so on, and (2) second order systems such as those encountered in hyperbolic heat conduction, hyperbolic second order systems such as elastodynamics and wave propagation, and so on. After space discretization using methods such as finite differences, finite volumes, finite elements, and the like, the consequent proper integration of the time continuous ordinary differential equations is extremely important. In particular, the physical quantities of interest may need to be mostly preserved and/or the equations should be optimally integrated so that there is minimal numerical dissipation, dispersion, algorithm overshoot, capture shocks without too much dissipation, solve stiff problems and enable the completion of the analysis, and so on. To-date, practical methods in most commercial and research software include the trapezoidal family (Euler forward/backward, Galerkin, and Crank Nicholson) for first order systems and the other counterpart trapezoidal family (Newmark family and variants with controllable numerical dissipation) for second order systems. For the respective first/second order systems, they are totally separate families of algorithms and are derived from altogether totally different numerical approximation techniques. Focusing on the class of the linear multistep (LMS) methods, algorithms by design was first utilized to develop GS4-2 framework for time integration of second order systems. We have also recently developed the GS4-1 framework for integrating first order systems. In contrast to all past efforts over the past 50 years or so, we present the formalism of a generalized unified framework, termed GS4 (generalized single step single solve), that unifies GS4-1 (first-order systems) and GS4-2 (second-order systems) frameworks for simultaneous use in both first and second order systems with optimal algorithms, numerical and order preserving attributes (in particular, second-order time accuracy) as well. The principal contribution emanating from such an integrated framework is the practicality and convenience of using the same computational framework and implementation when solving first and/or second order systems without having to resort to the individual framework. All that is needed is a single novel GS4-2 framework for either second- and/or first-order systems, and we show how to switch from one to the other for illustrative applications to thermo-mechanical problems influenced by first/second order systems, respectively.
\end{abstract}

Keywords: Dynamics; Time integration; Implicit methods; Finite element methods 